\title{
Reusing Integer Homology Information of Binary Digital Images ${ }^{\star}$
}

Rocío González-Díaz, Belén Medrano*^, Javier Sánchez-Peláez, and Pedro Real

\author{
Departamento de Matemática Aplicada I \\ Universidad de Sevilla, Seville, Spain \\ \{rogodi, belenmg, fjsp, real\}@us.es \\ http://www.us.es/gtocoma
}

\begin{abstract}
In this paper, algorithms for computing integer (co)homology of a simplicial complex of any dimension are designed, extending the work done in [1,2,3. For doing this, the homology of the object is encoded in an algebraic-topological format (that we call AM-model). Moreover, in the case of 3D binary digital images, having as input AM-models for the images $I$ and $J$, we design fast algorithms for computing the integer homology of $I \cup J, I \cap J$ and $I \backslash J$.
\end{abstract}

\section{Introduction}

Efficient algorithms for computing topological information are powerful tools in the fields of Data Mining, Pattern Recognition, Geometric Modeling and nD Digital Image Processing. Nevertheless, topological notions (such as the cup product on cohomology, cohomology operations, fundamental group, homotopy groups, etc) are hard to adapt into an n-dimensional discrete framework; and the number of available computational tools are limited. It is a fact that the cup product on cohomology is a topological invariant which contains more information than homology groups when we deal with an object of dimension greater than or equal to 3. Since cohomology is essentially an algebraic notion, it seems reasonable to encode it using a classical algebraic-topological cover: chain homotopy equivalences. In the setting of Simplicial Topology, we use here this extra algebraic-topological information (that we will define as an AM-model for a simplicial complex) to compute the cup product on integer cohomology as well as cohomological numbers derived from it, extending the work developed in [1,2]. Our computational approach follows the philosophy of the Effective Homology Theory developed by F. Sergeraert in 44,5. In particular, we prove that all the algorithms for computing integer homology based in the matrix reduction method to Smith normal form (for example $6,7,8,9$ ) can be translated to our setting with no extra computational cost in time. Finally, we successfully apply this computational algebraic topological approach to $3 \mathrm{D}$ binary digital images

\footnotetext{
* Partially supported by the PAICYT research project FQM-296 "Computational Topology and Applied Math" from Junta de Andalucía.

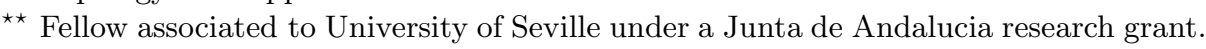


and we prove that a suitable extended notion of AM-model for binary 3D digital images can be reused under voxel-set operations (union, intersection and difference).

\section{Integer Homology, Chain Contractions and AM-Models}

In [9], an algorithm improving the efficiency of the classical integer reduction homology algorithm is described. Their technique is mainly based on the results of [8], in which a matrix reduction to integer Smith normal form is determined in an efficient way. There is no problem for translating this method to our framework since it consists in constructing a chain homotopy equivalence from the previously calculated Smith normal form, without additional computational cost. Moreover, our strategy of saving more algebraic information outperforms the previous algorithms for computing integer homology in several points such as: 1) cohomological features can be computed; 2) we can efficiently control the topological changes after addition or deletion of simplices.

First, we give a brief summary of concepts and notations. The terminology follows Munkres book 6. We will consider that the ground ring is $\mathbf{Z}$.

Simplicial Complexes. Considering an ordering on a vertex set $V$, a $q$-simplex with $q+1$ affinely independent vertices $v_{0}<\cdots<v_{q}$ of $V$ is the convex hull of these points, denoted by $\left\langle v_{0}, \ldots, v_{q}\right\rangle$. If $i<q$, an $i$-face of $\sigma$ is an $i$-simplex whose vertices are in the set $\left\{v_{0}, \ldots, v_{q}\right\}$. A simplex is maximal if it does not belong to any higher dimensional simplex. A simplicial complex $K$ is a collection of simplices such that every face of a simplex of $K$ is in $K$ and the intersection of any two simplices of $K$ is a face of each of them or empty. The set of all the $q$-simplices of $K$ is denoted by $K^{(q)}$. The dimension of $K$ is the dimension of the highest dimensional simplex in $K$.

Chains and Homology. Let $K$ be a simplicial complex. A $q$-chain $a$ is a formal sum of simplices of $K^{(q)}$. The $q$-chains form the qth chain group of $K$, denoted by $C_{q}(K)$. The boundary of a $q$-simplex $\sigma=\left\langle v_{0}, \ldots, v_{q}\right\rangle$ is the $(q-1)$ chain: $\partial_{q}(\sigma)=\sum_{i=0}^{q}(-1)^{i}\left\langle v_{0}, \ldots, \hat{v}_{i}, \ldots, v_{q}\right\rangle$, where the hat means that $v_{i}$ is omitted. By linearity, $\partial_{q}$ can be extended to $q$-chains. The collection of boundary operators connect the chain groups $C_{q}(K)$ into the chain complex $C(K): \cdots \stackrel{\partial_{2}}{\rightarrow}$ $C_{1}(K) \stackrel{\partial_{1}}{\rightarrow} C_{0}(K) \stackrel{\partial_{0}}{\rightarrow} 0$. An essential property is that $\partial_{q} \partial_{q+1}=0$. In a more general setting, a chain complex $\mathcal{C}$ is a sequence $\cdots \stackrel{d_{2}}{\longrightarrow} C_{1} \stackrel{d_{1}}{\longrightarrow} C_{0} \stackrel{d_{0}}{\longrightarrow} 0$ of abelian groups $C_{q}$ and homomorphisms $d_{q}$, such that for all $q, d_{q} d_{q+1}=0$. The set of all the homomorphisms $d_{q}$ is called the differential of $\mathcal{C}$. A $q$-chain $a \in C_{q}$ is called a $q$-cycle if $d_{q}(a)=0$. If $a=d_{q+1}\left(a^{\prime}\right)$ for some $a^{\prime} \in C_{q+1}$ then $a$ is called a $q$-boundary. Denote the groups of $q$-cycles and $q$-boundaries by $Z_{q}$ and $B_{q}$ respectively. Define the $q$ th homology group to be the quotient group $Z_{q} / B_{q}$, denoted by $H_{q}(\mathcal{C})$. We say that $a$ is a representative $q$-cycle of a homology generator $\alpha$ if $\alpha=a+B_{q}$. We denote $\alpha=[a]$. The $q$ th betti number $\beta_{q}$ is the rank of the free part of $H_{q}(\mathcal{C})$. Intuitively, $\beta_{0}$ is the number of components of 
connected pieces, $\beta_{1}$ is the number of independent "holes" and $\beta_{2}$ is the number of "cavities".

Chain Contractions. A chain contraction 10 of a chain complex $\mathcal{C}$ to a chain complex $\mathcal{C}^{\prime}$ is a set of three homomorphisms $(f, g, \phi)$ such that: $f: \mathcal{C} \rightarrow \mathcal{C}^{\prime}$ and $g: \mathcal{C}^{\prime} \rightarrow \mathcal{C}$ are chain maps; $f g$ is the identity map of $\mathcal{C}^{\prime} ;$ and $\phi: \mathcal{C} \rightarrow \mathcal{C}$ is a chain homotopy of the identity map $i d$ of $\mathcal{C}$ to $g f$, that is, $\phi \partial+\partial \phi=i d-g f$. In this case, $\mathcal{C}$ and $\mathcal{C}^{\prime}$ have isomorphic homology groups [6, p. 73].

AM-Models. An $A M$-model for a simplicial complex $K$ is the set $(C, M, f, g, \phi)$ where $C$ is a basis of $C(K), M$ is a subset of generators of $C(K)$ and $(f, g, \phi)$ is a chain contraction from $C(K)$ to $M(K)$ where $M(K)$ is the chain complex generated by $M$ with differential $\left.\partial\right|_{M(K)}$ such that in each dimension $q$, the matrix $A$ of the differential $\left.\partial_{q}\right|_{M(K)}$ coincides with its Smith normal form and satisfies that any non-null entry of $A$ is greater than 1 . Moreover, if the homology is free or the ground ring is a field, then $M(K)$ is isomorphic to the homology of $K$. It is necessary to emphasize that given a simplicial complex $K$, it is possible to define different AM-models for $K$ since the chain complex $M(K)$ and the morphisms $f, g$ and $\phi$ can admit different formulae.

A translation of the integer reduction homology algorithm in terms of chain contractions has been made in [11. Here we rewrite this work using a more algorithmic language. This algorithm consists in reducing the matrix $A_{q}$ of the boundary operator in each dimension $q$, to its Smith normal form $A_{q}^{\prime}$, relative to some basis $\left\{a_{1}, \ldots a_{r}\right\}$ of $C_{q}(K)$ and $\left\{e_{1}, \ldots, e_{s}\right\}$ of $C_{q-1}(K)$ such that $\left\{a_{\ell+1}, \ldots, a_{r}\right\}$ is a basis of $Z_{q}(K)$, and $\left\{\lambda_{1} e_{1}, \ldots, \lambda_{\ell} e_{\ell}\right\}$ is a basis of $B_{q-1}(K)$, pp. 56-61].

\section{Algorithm 1. Computing an AM-model for a Finite Simplicial Complex.}

INPUT: A simplicial complex $K$ of dimension $d$.

Initially: $C_{q}:=K^{(q)}, M_{q}:=K^{(q)}$ and $C_{q}^{\prime}=\{\}$ for $0 \leq q \leq d$, $f(\sigma):=\sigma, g(\sigma):=\sigma, \phi(\sigma):=0$ for every $\sigma \in K$.

For $q=1$ to $q=d$ do

Reduce the matrix $A_{q}$ of the boundary operator $\partial_{q}$ relative to the basis $C_{q}$ and $M_{q-1}$ to its Smith normal form $A_{q}^{\prime}$ relative to some basis $\left\{a_{1}, \ldots, a_{r}\right\}$ of $C_{q}$ and $\left\{e_{1}, \ldots, e_{s}\right\}$ of $M_{q-1}$ where:

$\partial_{q}\left(a_{i}\right)=e_{i}$, for $1 \leq i \leq t \leq \min (r, s)$;

$\partial_{q}\left(a_{i}\right)=\lambda_{i} e_{i}, \lambda_{i} \in \mathbf{R}$, for $t<i \leq \ell \leq \min (r, s)$;

and $\partial_{q}\left(a_{i}\right)=0$ for $\ell<i \leq r$.

Define $C_{q-1}:=C_{q-1}^{\prime} \cup\left\{e_{1}, \ldots, e_{s}\right\}, M_{q-1}:=\left\{e_{t+1}, \ldots, e_{s}\right\}$,

$C_{q}:=\left\{a_{1}, \ldots, a_{r}\right\}, C_{q}^{\prime}:=\left\{a_{1}, \ldots, a_{t}\right\}, M_{q}:=\left\{a_{t+1}, \ldots, a_{r}\right\}$,

$f\left(a_{i}\right):=0, f\left(e_{i}\right):=0$ and $\phi\left(e_{i}\right):=a_{i}$ for $1 \leq i \leq t$.

Output: The set $\left(C_{0} \cup \cdots \cup C_{d}, M_{0} \cup \cdots \cup M_{d}, f, g, \phi\right)$.

The following result shows that although $M(K)$ is not isomorphic to the homology of $K$, we can directly obtain the integer homology from it.

Theorem 2. Let $K$ be a finite simplicial complex of any dimension. The set $\left(C_{0} \cup \cdots \cup C_{d}, M_{0} \cup \cdots \cup M_{d}, f, g, \phi\right)$ defines an AM-model for $K$, being $C=$ 
$C_{0} \cup \cdots \cup C_{d}$ a basis of $C(K), M(K)$ the chain complex generated by $M=$ $M_{0} \cup \cdots \cup M_{d}$ and with differential $\left.\partial\right|_{M(K)}$, and $(f, g, \phi)$ a chain contraction from $C(K)$ to $M(K)$. Moreover, the integer homology of $K$ and integer homology generators can be directly obtained from $M$ and $\left.\partial\right|_{M(K)}$.

If $K$ has $m$ simplices, an AM-model for $K$ can be computed in time and storage $\mathcal{O}\left(\mathrm{m}^{3}\right)$.
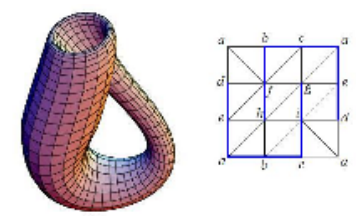

Fig. 1. The Klein bottle and a triangulation of it

Example 1. Consider the simplicial complex $K$ in Figure 1 whose underlying space is the Klein bottle [6, p. 283]. Running the algorithm above, we obtain that the vertex $\langle a\rangle$ belongs to $C_{0}$ and $M_{0}(K)$, the cycles $\alpha_{1}:=\langle a, b\rangle+\langle b, c\rangle-\langle a, c\rangle$ and $\alpha_{2}:=\langle a, d\rangle+\langle d, e\rangle-\langle a, e\rangle$ belong to $C_{1}$ and $M_{1}$; and the 2-chain consisting in the sum of all the triangles in $K, \beta:=-\langle a, b, f\rangle-\langle b, c, f\rangle+\langle a, c, g\rangle-\langle a, e, g\rangle+$ $\langle e, g, i\rangle-\langle e, d, i\rangle+\langle c, d, i\rangle-\langle a, c, d\rangle+\langle b, c, i\rangle+\langle a, b, h\rangle-\langle a, e, h\rangle-\langle e, d, f\rangle+$ $\langle a, d, f\rangle+\langle c, f, g\rangle-\langle f, g, h\rangle-\langle h, g, i\rangle-\langle b, h, i\rangle$, is an element of $C_{2}$ and $M_{2}$. The rest of the elements of $C_{0}$ are the boundaries of the 1-simplices marked in blue in Figure 1. These 1-simplices are also elements of $C_{1}$. Denote by $x$ one of these 1-simplices. The rest of the elements of $C_{1}$ are the boundaries of all the 2 -simplices except for $\langle f, g, h\rangle$. These 2-simplices belong to $C_{2}$. Denote by $y$ one of these 2-simplices. The images of the maps $(f, g, \phi)$ on the generators of $C(K)$ and $M(K)$ are described in the table below:

\begin{tabular}{c|c|c|c|c}
$C$ & $M$ & $f$ & $g$ & $\phi$ \\
\hline$\langle a\rangle$ & $\langle a\rangle$ & $\langle a\rangle$ & $\langle a\rangle$ & 0 \\
$\alpha_{1}$ & $\alpha_{1}$ & $\alpha_{1}$ & $\alpha_{1}$ & 0 \\
$\alpha_{2}$ & $\alpha_{2}$ & $\alpha_{2}$ & $\alpha_{2}$ & 0 \\
$\beta$ & $\beta$ & $\beta$ & $\beta$ & 0 \\
$x$ & & 0 & & 0 \\
$\partial x$ & & 0 & & $x$ \\
$y$ & & 0 & & 0 \\
$\partial y$ & & 0 & & $y$
\end{tabular}

Summing up, $M_{0}=\{\langle a\rangle\}, M_{1}=\left\{\alpha_{1}, \alpha_{2}\right\}$ and $M_{2}=\{\beta\}$. Moreover, $\partial(\langle a\rangle)=0$, $\partial\left(\alpha_{1}\right)=0, \partial\left(\alpha_{2}\right)=0$ and $\partial(\beta)=2 \alpha_{2}$. Therefore we obtain that $H_{0}(K) \simeq \mathbf{Z}$, $H_{1}(K) \simeq \mathbf{Z} \oplus \mathbf{Z} / \mathbf{Z} 2$ and representative cycles of the homology generators are $\langle a\rangle$ for $H_{0}(K), \alpha_{1}$ for the free part of $H_{1}(K)$ and $\alpha_{2}$ for the torsion part.

\section{Cohomology Computations with Integer Coefficients}

In this section, we extend the work done in [11,1,2] (with coefficients in a field) for computing cohomology features over the coefficient domain $\mathbf{Z}$. The interest 
for computing cohomology (the dual notion of homology) is that cohomology has an additional multiplicative structure, the cup product, from which we can derive finer invariants than homology. Observe that working with coefficients in a field, homology groups are free and isomorphic to cohomology groups. Nevertheless, working with coefficients in $\mathbf{Z}$, homology and cohomology of simplicial complexes can have torsion part and, in this case, they are not isomorphic.

Cochains and Cohomology. Let $\mathcal{C}$ be a chain complex. The cochain complex $\mathcal{C}^{*}$ in each dimension $q$ is the group of $q$-cochains with coefficients in $\mathbf{Z}, C^{q}=$ $\left\{c: C_{q} \rightarrow \mathbf{Z}\right.$ such that $c$ is a homomorphism $\}$. If $\left\{a_{1}, \ldots, a_{n}\right\}$ is a basis of $C_{q}$ then a basis of $C^{q}$ is $\left\{a_{1}^{*}, \ldots, a_{n}^{*}\right\}$, where $a_{i}^{*}: C_{q} \rightarrow \mathbf{Z}$ is given by $a_{i}^{*}\left(a_{i}\right)=1$ and $a_{i}^{*}\left(a_{j}\right)=0$ for $1 \leq i, j \leq n$ and $j \neq i$. For each $q$, the differential $d_{q+1}$ on $C_{q+1}$ induces the codifferential $\delta_{q}: C^{q} \rightarrow C^{q+1}$ via $\delta_{q}(c)=c d_{q+1}$, so that $\delta_{q}$ raises dimension by one. Define $Z^{q}$ to be the kernel of $\delta_{q}$ and $B^{q+1}$ to be its image. These groups are called the group of $q$-cocycles and $q$-coboundaries, respectively. Define the $q$ th cohomology group, $H^{q}(\mathcal{C})=Z^{q} / B^{q}$ for $q \geq 0$.

The following result shows that we can directly obtain the integer cohomology of $K$ from an AM-model for it. This assertion is not given in [11,1,2].

Theorem 3. Let $K$ be a finite simplicial complex of any dimension. Given an $A M$-model $(C, M, f, g, \phi)$ for $K$, the integer cohomology of $K$ and integer cohomology generators can be directly obtained from $M$ and $\left.\partial\right|_{M(K)}$.

Example 2. Consider the AM-model $(C, M, f, g, \phi)$ obtained in Example 1 for the simplicial complex $K$ whose underlying space is the Klein bottle. Starting from the chain complex $M(K)$ whose basis is $\left\{\langle a\rangle, \alpha_{1}, \alpha_{2}, \beta\right\}$ and differential $\left.\partial\right|_{M(K)}$, we construct in an straightforward way the cochain complex $M^{*}(K)$ whose basis is $\left\{\langle a\rangle^{*}, \alpha_{1}^{*}, \alpha_{2}^{*}, \beta^{*}\right\}$ and codifferential $\delta$ given by: $\delta\left(\langle a\rangle^{*}\right)=\left.\langle a\rangle^{*} \partial\right|_{M(K)}=0, \delta\left(\alpha_{1}^{*}\right)=\left.\alpha_{1}^{*} \partial\right|_{M(K)}=0, \delta\left(\alpha_{1}^{*}\right)=\left.\alpha_{2}^{*} \partial\right|_{M(K)}=2 \beta^{*}$, $\delta\left(\beta^{*}\right)=\left.\beta^{*} \partial\right|_{M(K)}=0$. Therefore we obtain that $H^{0}(K) \simeq \mathbf{Z}, H^{1}(K) \simeq \mathbf{Z}$ and $H^{2}(K) \simeq \mathbf{Z} / \mathbf{Z 2}$; and the generators are: $\langle a\rangle^{*}$ for $H^{0}(K), \alpha_{1}^{*}$ for $H^{1}(K)$ and $\beta^{*}$ for $H^{2}(K)$.

Cup Product. The cochain complex $C^{*}(K)$ is a ring with the cup product $\smile$ : $C^{p}(K) \times C^{q}(K) \rightarrow C^{p+q}(K)$ given by: $\left(c \smile c^{\prime}\right)\left(\left\langle v_{0}, \ldots, v_{p+q}\right\rangle\right)=c\left(\left\langle v_{0}, \ldots, v_{p}\right\rangle\right)$. $c^{\prime}\left(\left\langle v_{p}, \ldots, v_{p+q}\right\rangle\right)$. It induces an operation $\smile: H^{p}(K) \times H^{q}(K) \rightarrow H^{p+q}(K)$, via $[c] \smile\left[c^{\prime}\right]=\left[c \smile c^{\prime}\right]$, that is bilinear, associative, commutative up to a sign, independent of the ordering of the vertices of $K$ and homotopy-type invariant 6 , p. 289].

Working with coefficients in $\mathbf{Z} / \mathbf{Z} 2$, a new cohomology invariant called HB1 is obtained in [1,2]. The idea is to put into a matrix form the multiplication table of the cup product of cohomology generators of dimension 1 . The following algorithm compute HB1 working with integer coefficients. Assuming that $K$ has $m$ simplices, the complexity of this algorithm is $\mathcal{O}\left(m^{6}\right)$. This algorithm is an straightforward extension of that given in [1,2].

Algorithm 4. Algorithm for computing HB1 with integer coefficients. 
InPUT: An AM-model $(C, M, f, g, \phi)$ for a simplicial complex $K$. Let $\left\{\alpha_{1}, \ldots, \alpha_{p}\right\}$ and $\left\{\beta_{1}, \ldots, \beta_{m}\right\}$ be the set of 1 and 2 -cycles in $M$. For $i=1$ to $p$ do

For $j=i$ to $p$ do

For $k=1$ to $m$ do $b_{((i, j), k)}:=\left(\alpha_{i}^{*} f \smile \alpha_{j}^{*} f\right)\left(g\left(\beta_{k}\right)\right)$.

$\mathrm{HB} 1:=$ the rank of the 2D matrix of integers $B_{(p(p+1) / 2) \times m}=\left(b_{((i, j), k)}\right)$. OutPuT: The integer HB1.

The implementation of the algorithms described above working with coefficients in $\mathbf{Z} / \mathbf{Z 2}$ has been made by J. Sánchez-Peláez and P. Real. We have tested it on several 3D objects. We give here an example of the computation of the cohomology, cohomology generators and the invariant HB1.

Example 3. Consider the simplicial complex $T$ whose underlying space is showed in Figure 2 (on the left). It consists in 11847 simplices. The running time for computing an AM-model for $T$ and the homology of $T$ using a Pentium 4, 3.2 $\mathrm{GHz}, 1 \mathrm{~Gb} \mathrm{RAM}$ was 2 seconds. We obtain that $\beta_{0}=1, \beta_{1}=4$ and $\beta_{2}=3$. The running time for computing the cup product was 1.5 seconds. In Figure 2 (on the center), the 1 and 2-simplices on which the representative cocycles are non-null are drawing. The table on the right of Figure 2 shows the results of the cup product of any two cohomology generators of dimension 1 . Finally, HB1= 2 .
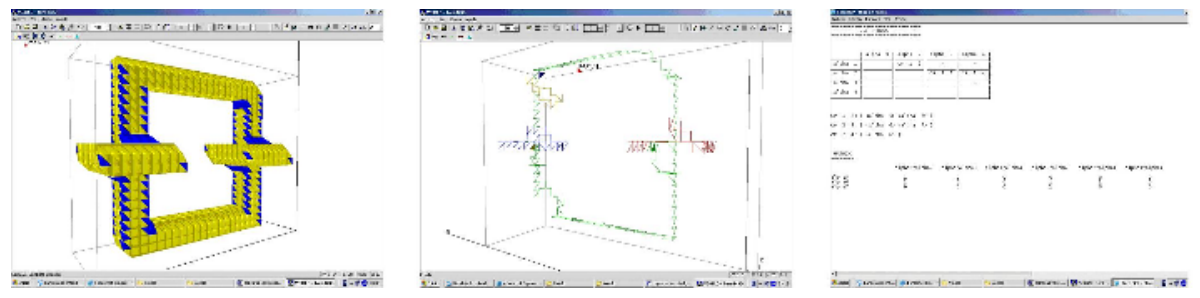

Fig. 2. The simplicial complex $T$, representative cocycles of the generators of $H^{1}(T)$ and $H^{2}(T)$ and the multiplication table of the cup product

\section{AM-Models for 3D Digital Images}

Three dimensional digital images are usually captured into the cubic grid or computed from 2D projections. There are, however, capturing techniques such as CT or MRI to produce images into other grids, such as the face-centered cubic (fcc) and the body-centered cubic (bcc) grids [12. An important issue in Digital Volume Processing is to design efficient algorithms for analysis and processing in these grids, since it is very easy to obtain data structures for the fcc and bcc grids. On the other hand, the only Voronoi adjacency relation on the bcc grid is the 14-adjacency. Using this adjacency, it is straightforward to associate to a digital image $I$, a unique simplicial complex $K(I)$ (up to isomorphism) with the 
same topological information as $I$. The $i$-simplices of $K(I)(i \in\{0,1,2,3\})$ are constituted by the different sets of $i$ mutually 14-neighbor black points in $I$.

Definition 1. Let I be a 3D binary digital image. An AM-model for I is defined as an AM-model for its simplicial representation $K(I)$.

Since simplicial complexes considered in this section are embedded in $\mathbf{R}^{3}$, their homology groups vanishes for dimensions greater than 3 and they are torsionfree for dimensions 0,1 and 2 (see [13, ch.10]). Therefore the chain complex $M(K(I))$ is isomorphic to the homology of $I$.

In the following table we present the running time for computing integer homology generators of the 3D digital images showed in figure 3. We have to say that these images have been created in a cubic grid. For these reason, we consider a special 14-adjacency in the cubic grid in the way that it is isomorphic to the bcc grid.

\begin{tabular}{|c|c|c|c|c|c|}
\hline Image $I$ & Number of voxels in $I$ & Time for computing & $\beta_{0}$ & $\beta_{1}$ & $\beta_{2}$ \\
\hline$A$ & 26308 & 50 seconds & 2 & 9 & 3 \\
$B$ & 31012 & 38 seconds & 138 & 419 & 13 \\
$C$ & 18842 & 27 seconds & 1 & 277 & 5 \\
\hline
\end{tabular}
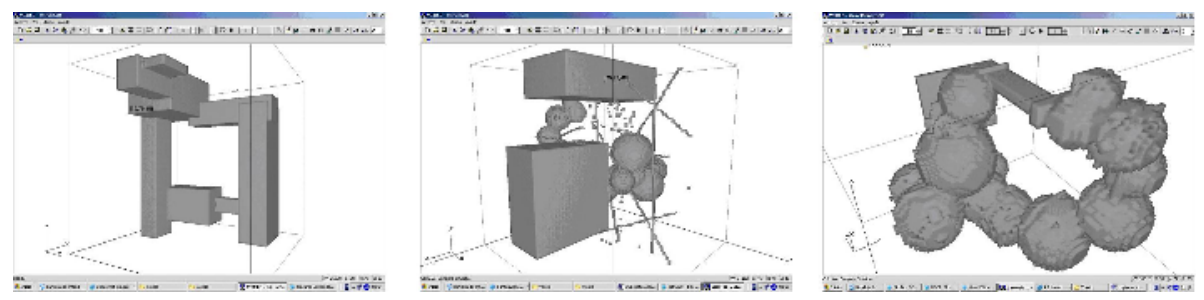

Fig. 3. The 3D digital images $A, B$ and $C$
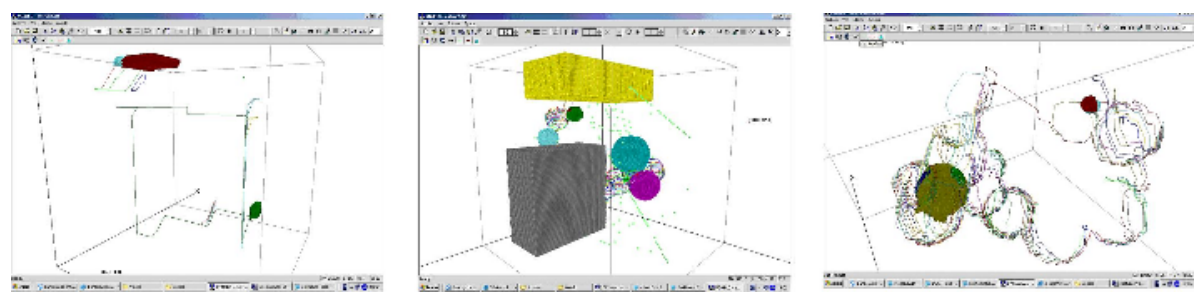

Fig. 4. Representative cycles of the homology generators of the images $A, B$ and $C$ 


\subsection{Computing "Good" Homology Generators}

In 14], algorithms for obtaining "optimal" generators of the first homology group are developed using Dijkstra's shortest path algorithm for any oriented 2-manifolds. Here, in the context of digital volumes we sketch some techniques for drawing "good" representative cycles of homology generators.

Given an AM-model $\left(K(I), M_{I}, f_{I}, g_{I}, \phi_{I}\right)$ for a $3 \mathrm{D}$ digital image $I$, we say that $x$ is the representative cycle of the generator $h \in M_{I}$ obtained by $g_{I}$ if $g_{I}(h)=x$. Our interest now is to get a new AM-model $\left(K(I), M_{I}^{\prime}, f_{I}^{\prime}, g_{I}^{\prime}, \phi_{I}^{\prime}\right)$ with "good" representative cycles of homology generators obtained by $g_{I}^{\prime}$. This means that each representative cycle obtained by $g_{I}^{\prime}$ belongs to the boundary of the image $\partial I$ (it is constituted by the set of black voxels in $I$ with a 14-neighbor white voxel). Moreover, it is required that in dimension 0 , it must be a vertex; in dimension 1, an elementary cycle (it is connected, each vertex is shared by exactly two edges and two consecutive edges can not belong to the same triangle in $K(I)$ ) and in dimension 2, an elementary cavity (it is a connected 2-cycle with exactly one white connected component inside and three triangles can not belong to the same tetrahedra).

Now, for obtaining good representative cycles of homology generators we can use the following new result.

Lemma 1. Let $(K, M, f, g, \phi)$ be an AM-model for a simplicial complex $K$. Let $h$ be a generator of $M$ and $x$ a chain in $C(K)$ such that $x=g(h)$. Let $x^{\prime}$ be a chain in $C(K)$ such that $\partial\left(x^{\prime}\right)=0$ and $f\left(x^{\prime}\right)=h$. Then, it is possible to define a new AM-model $\left.\left(K, M, f^{\prime}, g^{\prime}, \phi^{\prime}\right)\right)$ for $K$ such that $g^{\prime}(h):=x^{\prime}$ as follows: $g^{\prime}(h):=x^{\prime}$ and $g^{\prime}(z):=z$ if $z \neq h ; \phi^{\prime}(x):=\phi\left(x^{\prime}\right), \phi^{\prime}\left(x^{\prime}\right):=\phi(x)$ and $\phi^{\prime}(z):=\phi(z)$ for all $z \neq x^{\prime}, x$.

If we change the basis of $M(K)$ and/or the basis of $C(K)$, it is straightforward to obtain a new AM-model for $K$.

Now, suppose we have an AM-model $\left(K(I), M_{I}, f_{I}, g_{I}, \phi_{I}\right)$ for $I$ at hand. First of all, we compute an AM-model for $\partial I,\left(K(\partial I), M_{\partial I}, f_{\partial I}, g_{\partial I}, \phi_{\partial I}\right)$. If the elements of $M_{\partial I}$ are denoted by $\left\{\alpha_{1}, \ldots, \alpha_{n}\right\}$ then the set of representative cycles obtained by $g_{\partial I}$ is $S_{\partial I}=\left\{g_{\partial I}\left(\alpha_{1}\right), \ldots, g_{\partial I}\left(\alpha_{n}\right)\right\}$. Since all the homology generators of $I$ are homology generators of $\partial I$, find a subset $M_{I}^{\prime}$ of $\left\{f_{I} g_{\partial I}\left(\alpha_{1}\right), \ldots, f_{I} g_{\partial I}\left(\alpha_{n}\right)\right\}$, which is a basis of $M(K(I))$. Obtain the new AM-model $\left(K(I), M_{I}^{\prime}, f_{I}^{\prime}, g_{I}^{\prime}, \phi_{I}^{\prime}\right)$ for $I$ using Lemma 1. Now, denote by $S_{I}=\left\{c_{1}, \ldots, c_{m}\right\}$ the set of all the representative cycles obtained by $g_{I}^{\prime}$ which is a subset of $S_{\partial I}$. Decompose and replace each 0 -cycles in $S_{I}$ by its constitutive vertices, each 1-cycle by its elementary cycles and each 2-cycle by its elementary cavities. Let $M_{I}^{\prime \prime}:=\{\}$. For each cycle $s$ in $S_{I}$, if $\left\{f_{I}^{\prime}(s)\right\} \bigcup M_{I}^{\prime \prime}$ is a linearly-independent set then $M_{I}^{\prime \prime}:=\left\{f_{I}(s)\right\} \bigcup M_{I}^{\prime \prime}$; otherwise, $M_{I}^{\prime \prime}:=M_{I}^{\prime \prime}$. Obtain the new AM-model $\left(K(I), M_{I}^{\prime \prime}, f_{I}^{\prime \prime}, g_{I}^{\prime \prime}, \phi_{I}^{\prime \prime}\right)$ using Lemma 1]. Then, $\left(K(I), M_{I}^{\prime \prime}, f_{I}^{\prime \prime}, g_{I}^{\prime \prime}, \phi_{I}^{\prime \prime}\right)$ is an AM-model for $I$ with "good" representative cycles of homology generators of $I$.

\subsection{AM-Models After Adding or Deleting a Voxel}

Now, we study the problem of topologically controlling a digital image using AM-models when it suffers local changes (addition or deletion of one voxel). 
More concretely, we show how to compute an AM-model for a digital image when a voxel is added or deleted using the algebraic-topological information computed before. Assuming that $I$ has $m$ voxels, the complexity of the algorithms in this subsection is $\mathcal{O}\left(\mathrm{m}^{2}\right)$. The key idea for both algorithms is that when a $q$-simplex is added to or deleted from an AM-model of $K$, we only have to put into a Smith normal form the matrix of $\partial_{q}$ for obtaining the new AM-model. Moreover, take into account that adding or deleting a voxel $v$ of $I$ means to add or delete a set of simplices of $K(I \cup\{v\})$ having $v$ as a vertex. Since we work with simplicial complexes representing 3D digital images considering the 14-adjacency, the maximum number of simplices having $v$ as a vertex is 74 .

Let $(K, M, f, g, \phi)$ be an AM-model for a simplicial complex $K$. The differential of $M(K)$ is null since the homology is torsion free. Moreover, the value of all the possible non-null entries of the Smith normal form of the matrix of the differential of $C(K)$ in each dimension only can only be 1 .

AM-Models After Adding a Voxel. As we have mentioned before, the addition of a voxel $v$ to $I$ means the addition to $K(I)$ of all the simplices of $K(I \cup\{v\}) \backslash K(I)$. In each step of the process, one simplex is added. Assuming that $I$ has $m$ voxels, the following algorithm computes an AM-model for the image $I \cup\{v\}$ with integer coefficients in $\mathcal{O}\left(\mathrm{m}^{2}\right)$.

Algorithm 5. Incremental Algorithm for computing an AM-model for a $3 D$ Binary Digital Image.

InPUT: An AM-model $A M M_{I}=(K(I), M, f, g, \phi)$ for $I$ and a voxel $v \notin I$. Let $\left\{\sigma_{1}, \ldots, \sigma_{n}\right\} \quad(n \leq 74)$ be the ordered-by-increasing-dimension set of all the simplices of $K(I \cup\{v\}) \backslash K(I)$.

$K_{0}:=K(I)$.

For $i=1$ to $i=n$ do:

Let $q$ be the dimension of $\sigma_{i}$; let $C_{q}=\left\{a_{1}, \ldots, a_{r}\right\}$, $M_{q}=\left\{a_{t+1}, \ldots, a_{r}\right\}, C_{q-1}=\left\{e_{1}, \ldots, e_{s}\right\}$ and $M_{q-1}=\left\{e_{t+1}, \ldots, e_{s+1}\right\}$; let $\partial_{q}\left(a_{j}\right)=e_{j}$ for $1 \leq j \leq t$ and $\partial_{q}\left(a_{j}\right)=0$ for $t<j \leq \min (r, s)$;

let $\partial_{q}\left(\sigma_{i}\right)=\sum_{\ell=1}^{s} \lambda_{\ell} e_{\ell}$ where $\lambda_{\ell} \in \mathbf{R}$.

Define $a:=\sigma_{i}-\sum_{\ell=1}^{t} \lambda_{\ell} a_{\ell}$ and $C_{q}:=\left\{a_{1}, \ldots, a_{r}, a\right\}$.

If $\lambda_{\ell}=0$ for $\ell>t$ then $f(a):=a, g(a):=a, \phi(a):=0$ and $M_{q}:=\left\{a_{t+1}, \ldots, a_{r}, a\right\}$.

Else obtain the Smith normal form of the matrix of $\partial_{q}$ relative to some base $\left\{e_{1}, \ldots, e_{t}, e_{t+1}^{\prime}, \ldots, e_{s}^{\prime}\right\}$ of $C_{q-1}(K)$. Define $f(a):=0, \phi(a):=0, \phi\left(e_{t+1}^{\prime}\right):=a, f\left(e_{t+1}^{\prime}\right):=0$, $C_{q-1}:=\left\{e_{1}, \ldots, e_{t}, e_{t+1}^{\prime}, \ldots, e_{s}^{\prime}\right\}$ and $M_{q-1}:=\left\{e_{t+2}^{\prime}, \ldots, e_{s}^{\prime}\right\}$. $K_{i}:=K_{i-1} \cup\left\{\sigma_{i}\right\}$.

OutPut: An AM-model $\left(K_{n}, M, f, g, \phi\right)$ for $I \cup\{v\}$.

AM-Models After Deleting a Voxel from a 3D Digital Image. The deletion of a voxel $v$ from $I$ means the deletion from $K(I)$ of all the simplices having $v$ as a vertex. In each step of the process one simplex is deleted. Suppose that an AM-model for a digital image $I$ with $m$ voxeles has been computed and 
after this a voxel is deleted. The following algorithm computes an AM-model for the image $I \backslash\{v\}$ with integer coefficients in $\mathcal{O}\left(\mathrm{m}^{2}\right)$.

Algorithm 6. Decremental Algorithm for computing an AM-model for a $3 D$ Digital Image $I$.

INPUT: An AM-model $(K(I), M, f, g, \phi)$ for $I$ and a voxel $v \in I$.

Let $\left\{\mu_{1}, \ldots, \mu_{n}\right\} \quad(n \leq 72)$ be the ordered-by-decreasing-dimension set of all the simplices of $K(I)$ having $v$ as a vertex.

$K_{0}:=K(I)$.

For $i=1$ to $i=n$ do

Let $q$ be the dimension of $\sigma_{i}$; let $C_{q}=\left\{a_{1}, \ldots, a_{r}\right\}$, $M_{q}=\left\{a_{t+1}, \ldots, a_{r}\right\}, C_{q-1}=\left\{e_{1}, \ldots, e_{s}\right\}$ and $M_{q-1}=\left\{e_{t+1}, \ldots, e_{\ell+1}\right\}$;

let $\partial_{q}\left(a_{j}\right)=e_{j}$ for $1 \leq j \leq t$ and $\partial_{q}\left(a_{j}\right)=0$ for $t<j \leq \min (r, s)$

Find the element $a_{k} \in C_{q}$ such that

$C_{q}:=\left\{a_{1}, \ldots, \hat{a_{k}}, \ldots, a_{r}\right\}$ is a base of $C_{q}\left(K_{i-1} \backslash\left\{\sigma_{i}\right\}\right)$.

If $1 \leq k \leq t$ then

$M_{q-1}:=\left\{e_{k}, e_{t+1}, \ldots, e_{\ell+1}\right\}, f\left(e_{k}\right):=e_{k}, g\left(e_{k}\right):=e_{k}$ and $\phi\left(e_{k}\right):=0$.

Else $M_{q}:=\left\{a_{t+1}, \ldots, \hat{a_{k}}, \ldots, a_{r}\right\}$.

$K_{i}:=K_{i-1} \backslash\left\{\sigma_{i}\right\}$.

Output: An AM-model $\left(K_{m}, M, f, g, \phi\right)$ for $I \backslash\{v\}$.

\subsection{AM-Models Under Voxel-Set Operations on 3D Digital Images}

In this subsection, we efficiently reuse the AM-model information for digital images under voxel-set operations (union, intersection and difference).

Let $I$ and $J$ be two digital images. We will not consider these trivial cases: $I=\emptyset, J=\emptyset, I \cap J=\emptyset, I \subseteq J$ and $J \subseteq I$. Let $A M M_{I}:=\left(K(I), M_{I}, f_{I}, g_{I}, \phi_{I}\right)$ and $A M M_{J}:=\left(K(J), M_{J}, f_{J}, g_{J}, \phi_{J}\right)$ be AM-models for $I$ and $J$, respectively.

We give now the pseudocode of the algorithms we have developed for computing AM-models for $I \cup J, I \cap J$ and $I \backslash J$ starting from AM-models for $I$ and $J$. Denote by $F_{I}(J)=\left\{v_{1}, \ldots, v_{m}\right\}$ the set of all the voxels of $I \backslash J$ that are 14-neighbors of a voxel of $J$. Algorithm 7 is a common preprocessing to the three voxel-set operations treated here. In this algorithm, an AM-model for the image $I \backslash \operatorname{Fr}_{I}(J)$ is calculated.

\section{Algorithm 7. Preprocessing.}

InPUT: The AM-model $A M M_{I}$ and the set $F r_{I}(J)=\left\{v_{1}, \ldots, v_{m}\right\}$. $I_{m+1}:=I$.

For $i=m$ to $i=1$ do

apply Algorithm 6 to $v_{i}$ and the AM-model $\left(K\left(I_{i+1}\right), M_{I}, f_{I}, g_{I}, \phi_{I}\right)$.

$I_{i}:=I_{i+1} \backslash\left\{v_{i}\right\}$.

OutPut: An AM-model $\left(K\left(I_{1}\right), M_{I}, f_{I}, g_{I}, \phi_{I}\right)$ for $I \backslash F r_{I}(J)$.

For computing an AM-model for $I \cup J$, we first compute an AM-model for $(I \cup J) \backslash F r_{I}(J)$ using Algorithm 7 and after that we add the voxels of $F r_{I}(J)$ using Algorithm 5. 
Algorithm 8. Computing an AM-model for $I \cup J$.

InPUT: The AM-models $A M M_{I}$ for $I$ and $A M M_{J}$ for $J$ and the set $F r_{I}(J)$. Apply Algorithm 7 to $A M M_{I}$ and $\operatorname{Fr}_{I}(J)=\left\{v_{1}, \ldots, v_{m}\right\}$.

Define $I_{0}:=(I \cup J) \backslash F r_{I}(J)$;

$f(\mu):=f_{J}(\mu), \phi(\mu):=\phi_{J}(\mu)$ if $\mu \in K(J)$;

$f(\mu):=f_{I}(\mu), \phi(\mu):=\phi_{I}(\mu)$ if $\mu \in K_{0} \backslash K(J) ; M:=\operatorname{Imf}$;

$g(\alpha):=g_{J}(\alpha)$ if $\alpha \in M_{J}$ and $g(\alpha):=g_{I}(\alpha)$ if $\alpha \in M \backslash M_{J}$.

For $i=1$ to $i=m$ do

apply Algorithm [5 to $v_{i}$ and the AM-model $\left(K\left(I_{i-1}\right), M, f, g, \phi\right)$.

$I_{i}:=I_{i-1} \cup\left\{v_{i}\right\}$.

OutPut: an AM-model $\left(K\left(I_{m}\right), M, f, g, \phi\right)$ for $I \cup J$.

Algorithm 7 is also the essential step for computing an AM-model for $I \cap J$.

Algorithm 9. Computing an AM-model for $I \cap J$.

InPUT: The AM-model $A M M_{I}$ for $I$ and the set $F r_{I}(J)$.

Apply Algorithm 7 to $A M_{I}$ and $\operatorname{Fr}_{I}(J)$.

Define $f(\mu):=f_{I}(\mu)$ and $\phi(\mu):=\phi_{I}(\mu)$ if $\mu \in K(I \cap J)$;

$M:=\operatorname{Imf}$ and $g(\alpha):=g_{I}(\alpha)$ if $\alpha \in M$.

Output: an AM-model $(K(I \cap J), M, f, g, \phi)$ for $I \cap J$.

For computing an AM-model for $I \backslash J$, we first apply Algorithm 7. Second, we consider the voxels that are in $I \backslash\left(J \cup F r_{I}(J)\right.$. Finally, we add the voxels of $\operatorname{Fr}_{I}(J)$ using Algorithm 5.

Algorithm 10. Computing an AM-model for $I \backslash J$.

InPUT: The AM-model $A M M_{I}$ for $I$ and the set $F r_{I}(J)$.

Apply Algorithm 7 to $A M M_{I}$ and $\operatorname{Fr}_{I}(J)=\left\{v_{1}, \ldots, v_{m}\right\}$.

Define $I_{0}:=I \backslash\left(J \cup F r_{I}(J) ; f(\mu):=f_{I}(\mu)\right.$ and $\phi(\mu):=\phi_{I}(\mu)$ if $\mu \in K\left(I_{0}\right)$ $M:=\operatorname{Imf} ; g(\alpha):=g_{I}(\alpha)$ if $\alpha \in M$.

For $i=1$ to $i=m$ do

apply Algorithm [5 to $v_{i}$ and the AM-model $\left(K\left(I_{i-1}\right), M, f, g, \phi\right)$.

$I_{i}:=I_{i-1} \cup\left\{v_{i}\right\}$.

OutPuT: an AM-model $\left(K\left(I_{m}\right), M, f, g, \phi\right)$ for $I \backslash J$.

\section{Comments}

The algebraic-topological representation of simplicial complexes of any dimension showed here, allows us to compute topological invariants derived from the integer cohomology ring. Moreover, we give a positive answer to the problem of efficiently reusing AM-models for determining homological information of new $3 \mathrm{D}$ binary digital images constructed from the previous ones using voxel-set operations.

There is considerable scope for further research: 1) To compute cohomology operations or homotopy groups of simplicial complexes using AM-models. 
2) To suitably extend our method to $\mathrm{nD}$ binary digital images in any grid using simplicial analogous techniques [15, 16, 17, 18.

Potential applications of our particular method in computer vision and digital image processing involving not only 3D object but also higher dimensional structures can be encountered in Medical Imaging and Object Modeling. Our method seems to be especially well adapted to segmentation under topological constraints and elimination of small topological noise.

\section{References}

1. González-Díaz R., Real P.: Towards Digital Cohomology. DGCI 2003, LNCS, Springer 2886 (2003) 92-101

2. González-Díaz R., Real P.: On the Cohomology of 3D Digital Images. Discrete Applied Math 147 (2005) 245-263

3. Gonzalez-Diaz R., Medrano B., Real P., Sánchez-Peláez J.: Algebraic Topological Analysis of Time-Sequence of Digital Images. Lecture Notes in Computer Science 139 (2005) 208-219

4. Sergeraert F.: Homologie effective. I, II. C. R. Acad. Sci. Paris Sér. I Math. 304 (1987), no. 11, 279-282, no. 12, 319-321

5. Sergeraert F.: The computability problem in algebraic topology. Adv. Math. 104 (1994), no. 1, 1-29

6. Munkres J.R.: Elements of Algebraic Topology. Addison-Wesley Co. 1984

7. Agoston M.K.: Algebraic Topology, a first course. Marcel Dekker Ed., 1976

8. Dumas J.G., Saunders B.D., Villad G.: On efficient sparse integer matrix Smith normal form computations. Journal of Symbolic Computation 32 (2001) 71-99

9. Peltier S., Alayrangues S., Fuchs L., Lachaud J.: Computation of homology groups and Generators. LNCS, 3429 (2005) 195-205

10. MacLane S.: Homology. Classic in Math., Springer-Verlag, 1995

11. González-Díaz R., Real P.: Computation of Cohomology Operations on Finite Simplicial Complexes. Homology, Homotopy and Applications 5 (2) (2003) 83-93

12. Herman, G.T.: Geometry of Digital Spaces. Birkhauser, Boston (1998).

13. Alexandroff P., Hopf H.: Topologie I. Springer, Berlin 1935

14. Erickson J., Whittlesey K.: Greedy Optimal Homotopy and Homology Generators. Proc. of the sixteenth annual ACM-SIAM symposium on Discrete algorithms (2005) 1038 - 1046

15. Kong T.Y.: A Digital Fundamental Group. Computer Graphics 13 (1989) 159-166

16. Kong T.Y., Roscoe A.W., Rosenfeld A.: Concepts of Digital Topology. Topology and its Applications 46 (1992) 219-262

17. Khalimsky E.D., Kopperman R.D., Meyer P.R.: Computer Graphics and Connected Topologies on Finite Ordered Sets. Topology and Appl. 36 (1990) 1-17

18. Ayala R., Domínguez E., Francés A.R., Quintero A.: Homotopy in Digital Spaces. DGCI 2000. LNCS, Springer-Verlag 1953 (2000) 3-14 